\title{
Characterization of pR18, a novel rolling-circle replication plasmid from Lactobacillus plantarum
}

\begin{abstract}
Lactobacillus plantarum PA18, a strain originally isolated from the leaves of Pandanus amaryllifolius, contains a pR18 plasmid. The pR18 plasmid is a $3211 \mathrm{bp}$ circular molecule with a $\mathrm{G}+\mathrm{C}$ content of $35.8 \%$. Nucleotide sequence analysis revealed two putative open reading frames, ORF1 and ORF2, in which ORF2 was predicted (317 amino acids) to be a replication protein and shared 99\% similarity with the Rep proteins of pLR1, pLD1, pC30il, and pLP2000, which belong to the RCR pC194/pUB110 family. Sequence analysis also indicated that ORF1 was predicted to encode linA, an enzyme that enzymatically inactivates lincomycin. The result of Southern hybridization and mung bean nuclease treatment confirmed that pR18 replicated via the RCR mechanism. Phylogenetic tree analysis of pR18 plasmid proteins suggested that horizontal transfer of antibiotic resistance determinants without genes encoding mobilization has not only occurred between Bacillus and Lactobacillus but also between unrelated bacteria. Understanding this type of transfer could possibly play a key role in facilitating the study of the origin and evolution of lactobacillus plasmids. Quantitative PCR showed that the relative copy number of pR18 was approximately 39 copies per chromosome equivalent.
\end{abstract}

Keyword: Lactobacillus plantarum; Cryptic plasmid; Rep; linA; qPCR 